\title{
Safe and stable noninvasive focal gene delivery to the mammalian brain following focused ultrasound
}

\author{
Mihaela A. Stavarache, MD, ${ }^{1}$ Nicholas Petersen, BA, ${ }^{1}$ Eric M. Jurgens, BS, ${ }^{1}$ Elizabeth R. Milstein, ${ }^{1}$ \\ Zachary B. Rosenfeld, BS, ${ }^{1}$ Douglas J. Ballon, PhD, ${ }^{2}$ and Michael G. Kaplitt, MD, PhD'
}

${ }^{1}$ Laboratory of Molecular Neurosurgery, Department of Neurological Surgery, and ${ }^{2}$ Citigroup Biomedical Imaging Center, Department of Radiology, Weill Cornell Medical College, New York, New York

OBJECTIVE Surgical infusion of gene therapy vectors has provided opportunities for biological manipulation of specific brain circuits in both animal models and human patients. Transient focal opening of the blood-brain barrier (BBB) by MR-guided focused ultrasound (MRgFUS) raises the possibility of noninvasive CNS gene therapy to target precise brain regions. However, variable efficiency and short follow-up of studies to date, along with recent suggestions of the potential for immune reactions following MRgFUS BBB disruption, all raise questions regarding the viability of this approach for clinical translation. The objective of the current study was to evaluate the efficiency, safety, and long-term stability of MRgFUS-mediated noninvasive gene therapy in the mammalian brain.

METHODS Focused ultrasound under the control of MRI, in combination with microbubbles consisting of albumin-coated gas microspheres, was applied to rat striatum, followed by intravenous infusion of an adeno-associated virus serotype 1/2 (AAV1/2) vector expressing green fluorescent protein (GFP) as a marker. Following recovery, animals were followed from several hours up to 15 months. Immunostaining for GFP quantified transduction efficiency and stability of expression. Quantification of neuronal markers was used to determine histological safety over time, while inflammatory markers were examined for evidence of immune responses.

RESULTS Transitory disruption of the BBB by MRgFUS resulted in efficient delivery of the AAV1/2 vector to the targeted rodent striatum, with $50 \%-75 \%$ of striatal neurons transduced on average. GFP transgene expression appeared to be stable over extended periods of time, from 2 weeks to 6 months, with evidence of ongoing stable expression as long as 16 months in a smaller cohort of animals. No evidence of substantial toxicity, tissue injury, or neuronal loss was observed. While transient inflammation from BBB disruption alone was noted for the first few days, consistent with prior observations, no evidence of brain inflammation was observed from 2 weeks to 6 months following MRgFUS BBB opening, despite delivery of a virus and expression of a foreign protein in target neurons.

CONCLUSIONS This study demonstrates that transitory BBB disruption using MRgFUS can be a safe and efficient method for site-specific delivery of viral vectors to the brain, raising the potential for noninvasive focal human gene therapy for neurological disorders.

https://thejns.org/doi/abs/10.3171/2017.8.JNS17790

KEYWORDS adeno-associated virus; gene therapy; focused ultrasound; blood-brain barrier; inflammation; rodent

$\mathrm{G}$ ENE therapy has long held promise as a potentially groundbreaking method for improving a variety of complex disorders. The brain has been a major focus of translational gene therapy research, with several human clinical trials showing safety and evidence of efficacy for Parkinson's disease, ${ }^{1,22,24,25}$ Alzheimer's disease, ${ }^{40,46,47}$ and a variety of neurogenetic disorders. Due to the pres- ence of the blood-brain barrier (BBB) and its highly selective permeability, ${ }^{1,27}$ the only current means for efficient delivery of viral vectors to specific regions in the human brain have been through invasive direct injection. Not only does this method carry the attendant risks of invasive surgery, but also efficient distribution of gene therapy agents throughout a target area can be difficult to confirm with

ABBREVIATIONS AAV = adeno-associated virus; $A A V 1 / 2=A A V$ serotype 1/2; $B B B=$ blood-brain barrier; $B S A=$ bovine serum albumin; $D A B=3,3^{\prime}$-diaminobenzidine; $\mathrm{DAPI}=$ 4',6-diamino-2-phenylindole; Gd-DTPA = gadopentetate dimeglumine; GFP = green fluorescent protein; MRgFUS = MR-guided focused ultrasound; PBS = phosphate-buffered saline; rAAV = recombinant AAV; RF = radiofrequency; TBST = Tris-buffered saline with Triton.

SUBMITTED March 28, 2017. ACCEPTED August 28, 2017.

INCLUDE WHEN CITING Published online April 27, 2018; DOI: 10.3171/2017.8.JNS17790. 
traditional infusion methods. Newer approaches have been tested that permit monitoring of contrast spread during infusion as a surrogate for viral vector distribution utilizing specialized catheter systems with intraoperative MRI methodology., ${ }^{9,41}$

To reduce surgical risks and avoid the complexities of direct infusion, noninvasive approaches have been explored to permit intravenous delivery of viral vectors into the brain. Use of an osmotic agent such as mannitol has long been known to transiently open the BBB, permitting delivery of a variety of agents to the brain, including viral vectors. ${ }^{5,34,39,42}$ However, systemic administration of mannitol induces widespread opening of the BBB, precluding target-specific gene expression. Selective intraarterial delivery of BBB disruption agents could provide more targeted gene delivery and cover larger brain areas, but variability in the vascular supply of various important deep-brain structures creates challenges for reproducible delivery between individuals. ${ }^{10}$

An alternative to chemical delivery is mechanical disruption of the BBB. One approach that has recently gained increasing interest is MR-guided focused ultrasound (MRgFUS). ${ }^{15,16,29}$ This approach involves focused delivery of ultrasound to a target region, and high-frequency MRgFUS has been used in human patients to create targeted brain lesions to treat essential tremor and pain. ${ }^{6,7,17}$ Use of MRgFUS at lower frequency, in combination with microbubble-mediated cavitation, has been shown to focally open the BBB to facilitate transfer of drugs, ${ }^{45}$ antibodies ${ }^{18}$ and nanoparticles ${ }^{32}$ from the blood stream to the brain parenchyma. This has also been used for noninvasive gene delivery to the rodent brain., ${ }^{2,13,14,43,50}$ These reports have shown successful transfer of adeno-associated virus (AAV) vectors from the blood stream to the brain following MRgFUS-mediated BBB opening with variable efficiencies and with transgene expression evaluated for relatively short periods following delivery. ${ }^{2,13,43,50}$ Because the goal of gene delivery is long-term neuronal modification, long-term expression following MRgFUS-mediated gene delivery remains to be confirmed. This is particularly important because potential immune-mediated loss of gene expression or transduced cells, due to exposure of the brain to the immune system following BBB disruption, might not fully manifest until later time points, as has been observed in some gene transfer studies outside of the brain., 3,4,23,30,48 Furthermore, the potential for provoking inflammation in brain parenchyma following the application of focused ultrasound has only been examined up to 2 weeks following either MRgFUS BBB disruption alone $^{18,21}$ or delivery of AAV vectors. ${ }^{43,49}$ The potential consequences of long-term brain exposure to a potential immunogenic viral vector following BBB disruption remain unknown.

In this study we report that MRgFUS-mediated BBB disruption can lead to efficient delivery and wide distribution of AAV vectors to the intended brain target in rodents. We further demonstrate that gene expression is stable over extended periods of time (6-16 months), comparable to what we have historically observed with direct infusion. Finally, while we observed a mild initial inflammatory response for the first 2 days following BBB disruption, we did not observe evidence of inflammation over the long term, and there was no evidence of behavioral or histological toxicity at any time point.

\section{Methods}

\section{Recombinant AAV Vectors}

AAV serotype 1/2 (AAV1/2) hybrid vector stocks, encoding the reporter gene green fluorescent protein (GFP) under the control of the CAG promoter, were prepared by packaging the plasmids into AAV particles containing capsid proteins for both AAV1 and AAV2 to create AAV1/2.GFP using a helper-free plasmid transfection system that we have described previously. ${ }^{20,31}$ Vectors were purified using heparin affinity chromatography and dialyzed against phosphate-buffered saline (PBS). Recombinant AAV (rAAV) titers were determined by quantitative polymerase chain reaction using cytomegalovirus enhancer-specific primers and adjusted to $10^{9}$ vector genomic particles (vg) per microliter.

\section{Animal Preparation and Experimental Design}

All animal procedures were approved by the Institutional Animal Care and Use Committee of Weill Cornell Medical College and followed NIH guidelines. Ten-weekold male Sprague Dawley rats (250-300 g; Charles River Laboratories) were used in all studies. Four rats were used to assess the efficiency and safety of unilateral MRgFUSmediated AAV1/2.GFP delivery to the striatum at 3 weeks after sonication. Another group of 15 rats underwent a similar procedure but were killed at different time points: 2 weeks $(n=3), 2$ months $(n=4), 6$ months $(n=6)$, and 16 months $(n=2)$. The brains and several organs (liver, lung, and heart) were then harvested and processed for histological analysis (Supplemental Fig. 1).

\section{MRgFUS and Viral Vector Delivery}

Animals were anesthetized using a ketamine $(90 \mathrm{mg} /$ $\mathrm{kg})$ and xylazine $(4 \mathrm{mg} / \mathrm{kg})$ cocktail. A 22-G intravenous catheter (BD InsyteAutoguard) was inserted into the lateral tail vein for substance administration during experiments. After scalp shaving, animals were secured in a supine position on the focused ultrasound system and the head was coupled with the degassed water tank holding the transducer. The spherically focused transducer (7- $\mathrm{cm}$ diameter, $\mathrm{f \#}=0.8)$ was driven by a computer-controlled function generator (33220A Agilent Function/Arbitrary $20 \mathrm{MHz}$ waveform generator; Agilent Technologies) and a 43-dB radiofrequency (RF) power amplifier (FUS Instruments).

Before sonication, MRI was performed with a 3.0-T GE scanner, using a $4 \times 7-\mathrm{cm}$ RF surface coil. T2-weighted axial images (10 slices) perpendicular to the direction of the ultrasound beam propagation were acquired before sonication to calculate the coordinates of the target. The transducer was then moved to the desired position using a motorized 3-axis positioning system (FUS Instruments). The striatum was sonicated in 4 points, $1.5 \mathrm{~mm}$ apart. Assuming a $49 \%$ loss of ultrasound power due to attenuation through the rat skull, ${ }^{44}$ an estimated in situ rarefactional pressure of $0.97 \mathrm{MPa}$ was applied at the sonication points, with a $1-\mathrm{Hz}$ pulse repetition frequency, $10-\mathrm{msec}$ burst 
length, and 200-second total sonication time. The cocktail of rAAV and Optison microspheres (Perflutren ProteinType A microspheres, mean size between 3 and $4.5 \mu \mathrm{m}$, dose between $0.4 \times 10^{8}$ microbubbles $/ \mathrm{kg}$ and $-0.64 \times 10^{8}$ microbubbles/kg; GE Healthcare Life Sciences) was administered simultaneously with sonication through the tail vein catheter, followed by MRI contrast agent Magnevist (gadopentetate dimeglumine [Gd-DTPA], $0.4 \mathrm{ml} / \mathrm{kg}$; Bayer). T1-weighted images (7 slices) were collected at the conclusion of sonication to monitor the degree of the BBB opening based on contrast extravasation. The slice thickness was $0.8 \mathrm{~mm}$, with a spacing of $0.2 \mathrm{~mm}$.

\section{Immunohistochemistry and Histology}

Rats were deeply anesthetized with sodium pentobarbital $(150 \mathrm{mg} / \mathrm{kg})$ and transcardially perfused with ice-cold $0.1 \mathrm{M}$ heparinized PBS (pH 7.4) followed by 4\% (weight/ volume) buffered paraformaldehyde solution. The brains, liver, heart, and lungs were removed, postfixed in the same fixative solution for 24 hours, and subsequently immersed in $30 \%$ (weight/volume) sucrose cryoprotective solution at $4^{\circ} \mathrm{C}$. The brains were frozen and sectioned serially $(6$ series per brain) into $40-\mu \mathrm{m}$-thick sections in the coronal plane on an AO Spencer 860 sliding microtome. The organs were embedded in agar solution and $40-\mu \mathrm{m}$-thick sections were cut on a Leica VT1200 vibratome for histological analysis.

To determine the extent of the area transduced by GFP, an entire series of brain coronal sections per each animal was rinsed in Tris-buffered saline with $0.1 \%$ Triton (TBST). Following the quenching of endogenous peroxides with a $0.3 \%$ solution of hydrogen peroxide in TBST, sections were incubated in blocking solution (3\% bovine serum albumin [BSA] and $2 \%$ goat serum in TBST) for 1 hour at room temperature and then for 24 hours at $4^{\circ} \mathrm{C}$ with a rabbit polyclonal anti-GFP antibody (Abcam, ab290, 1:4000). The following day, sections were rinsed in TBST before a 1-hour incubation with biotinylated secondary antibodies. Following several washes, sections were incubated with Vectastain Elite ABC kit (1:500) in TBST for 1 hour. Staining was visualized using a 3,3'-diaminobenzidine $(\mathrm{DAB})$ peroxidase substrate solution.

Identification of the neurons transduced by GFP was performed using an immunofluorescence protocol. Sections were incubated in blocking solution (3\% BSA and $2 \%$ goat serum in TBST) for 1 hour at room temperature and then for 24 hours at $4^{\circ} \mathrm{C}$ with a rabbit polyclonal anti-GFP (Abcam, ab290, 1:4000), and mouse monoclonal anti-NeuN (neuronal marker beta-tubulin III; Abcam, AB104224, 1:1000) antibodies. The following day, sections were rinsed and incubated in goat anti-rabbit Alexa Fluor 488 and goat anti-mouse Alexa Fluor 594-conjugated secondary antibodies (Life Technologies), and nuclei were stained with 4',6-diamino-2-phenylindole (DAPI; Invitrogen, 1:10,000). Hematoxylin and eosin staining was used to evaluate intact cells and tissue integrity.

For detection of inflammatory markers, sections were incubated with mouse monoclonal anti-Ibal (Millipore, MABN92, 1:500) and rabbit monoclonal anti-GFAP (Abcam, ab7260, 1:1000) antibodies and the staining was visualized with goat anti-mouse Alexa Fluor 488 and goat anti-rabbit Alexa Fluor 594-conjugated secondary antibodies (Life Technologies). The nuclei were stained with DAPI (Invitrogen, 1:10,000).

\section{Image Analysis and Quantitative Analysis of GFP-Positive Area}

Quantitative analysis of the GFP-transduced striatum was performed using ImageJ Fiji software. Z-stacks from 4 sections per animal were collected at $10 \times$ magnification using an Olympus BX61 upright microscope, and cells were quantified from 20 random fields taken from a predefined region of interest within the sonicated striatum $(100 \times 100 \times 40-\mu \mathrm{m}$ depth $)$. The region of interest was kept constant between animals to permit between-animal comparison. To determine the proportion of GFP-transduced neuronal and nonneuronal cells, colocalization of GFP and NeuN was analyzed using immunofluorescence microscopy. Neuronal cell transduction rate was calculated by expressing GFP-positive neuronal cells as a percentage of the total number of neurons in the analyzed area. To quantify the distribution of neuronal and nonneuronal cells among GFP-positive cells, both GFP-positive neuronal cells and GFP-positive nonneuronal cells were expressed as a percentage of total GFP-transduced cells.

\section{Statistical Analysis}

A 2-tailed t-test was used for statistical comparison of all groups. All data are expressed as the mean value with standard error of the mean (SEM). When the $p$ value was $<0.05$, the difference was considered statistically significant.

\section{Results \\ MRgFUS-Facilitated, AAV1/2-Mediated GFP Gene Transduction of Rat Striatum}

To first evaluate the efficiency of our system to safely and transiently disrupt the BBB in the striatum, 3 rats underwent unilateral striatal MRI-guided 4-point sonication. A postsonication T1-weighted MR image demonstrated local extravasation of Gd-DTPA into the local brain tissue, and confirmed BBB disruption (Fig. 1A). Gd-DTPA extravasation overlapped the striatal area selected on the T2-weighted presonication MRI. Three weeks later, immunohistological analysis confirmed GFP transgene expression in the sonicated striatum, in both neuronal and nonneuronal cells, while no signal was detected on the contralateral side of the brain (Fig. 1B and C, Supplemental Fig. 2). DAB chromogenic staining of GFP transgene in a series of sections showed an extensive and efficient anteroposterior and dorsomedial GFP transduction of the targeted striatum within the volume of tissue subject to sonication (Fig. 1C). In addition, hematoxylin and eosin staining revealed no evidence of tissue damage in the sonicated area (Supplemental Fig. 2).

\section{MRgFUS-Facilitated, AAV1/2-Mediated GFP Expression: Efficiency and Stability Over an Extended Period}

Although long-term stability of AAV-mediated gene expression has been well established in the brain following 

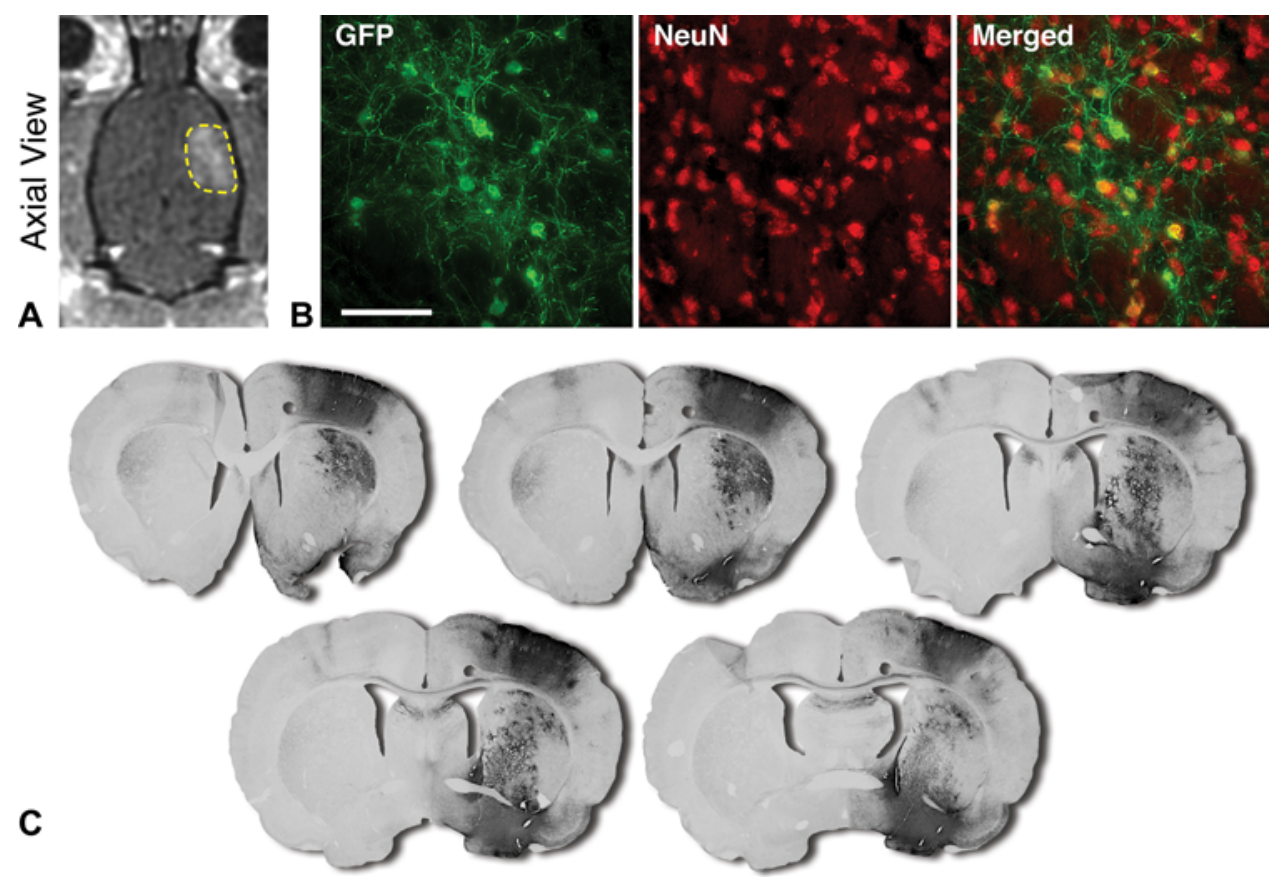

FIG. 1. MRgFUS facilitates AAV-mediated gene delivery to the brain. A: Gd-DTPA-enhanced T1-weighted images collected after sonication showed disruption of the BBB and Gd-DTPA extravasation in brain parenchyma (dashed line). B: The brain tissue was harvested 3 weeks after sonication and the colocalization of GFP and NeuN was visualized using immunostaining. Bar $=50$ $\mu \mathrm{m}$. C: DAB visualization of GFP transduction from serial sections centered on the targeted area (serial sections through the center of the targeted points).

direct infusion, factors such as exposure to the immune system following BBB disruption could influence longevity of gene expression and survival of transduced neurons. GFP immunostaining with DAB-peroxidase substrate performed in rat tissue collected 6 months after sonication revealed long-term expression of GFP transgene through extended brain parenchyma (Video 1).

VIDEO 1. Clip showing a 3D model of an MRgFUS-mediated GFPtransduced rat brain. This 3D model was created from brain sections immunostained for GFP expression and harvested 6 months after MRgFUS in an animal that underwent 4-point sonication. The $3 \mathrm{D}$ reconstructed rat brain revealed the volume of Gd-DTPA extravasated into brain parenchyma following the BBB opening. Copyright Michael Kaplitt. Published with permission. Click here to view.

Triple immunolabeling for the GFP transgene with NeuN and DAPI allowed us to quantify transduced neurons and nonneuronal cells from 2 weeks to 6 months following MRgFUS-facilitated AAV1/2.GFP delivery (Fig. 2). Quantification of GFP-positive neurons from the total number of neurons revealed transduction of between $50 \%$ and $74 \%$ of neurons within the analyzed region. A lower rate of transduction was observed at 2 weeks (50\%), with higher transduction rates noted at 2 months (74\%) and 6 months (63\%; Fig. 2B). This could be consistent with the known pattern of AAV-mediated transduction, which generally increases over several weeks following delivery, but the relatively small number of animals in the 2-week group could also have influenced this variation. Analysis of the type of GFP-transduced cells revealed that neurons represented $86 \%-98 \%$ of total GFP-expressing cells (Fig. $2 \mathrm{C})$, again consistent with our previous experience with direct infusion of this particular AAV serotype. Immunohistological analysis of GFP-NeuN colocalization of 2 animals that were sacrificed at 16 months after MRgFUSfacilitated AAV-mediated GFP delivery revealed a similar prevalence of GFP neurons in the transduced striatum (Fig. 3, Supplemental Fig. 4). These two subjects were not part of the cohort of animals included in the time course study, but had been treated much earlier during the pilot period, and therefore it is difficult to directly compare cell numbers in these 2 animals compared with the rest of the time course cohort. Nonetheless, these data show that there is demonstrable ongoing expression 16 months after MRgFUS-mediated gene delivery. While the focus of our transducer was on the striatum, the nature of the conical ultrasound beam from the single curved transducer resulted in considerable BBB disruption in the overlying cortex. Transduction of the overlying cortex was similar to what we observed with the striatum, suggesting that MRgFUS is capable of efficient and stable gene delivery to different brain regions (Supplemental Figs. 5 and 6). Finally, neuronal quantification revealed no evidence of neuronal loss over time in either the sonicated striatum or cortex and no difference in these regions compared with the corresponding nonsonicated hemisphere (Supplemental Fig. 6).

\section{Transient Peripheral MRgFUS-Facilitated GFP Gene Transduction}

To noninvasively deliver AAV into the brain via MRgFUS, vectors were infused intravenously, which could also result in transduction of peripheral organs depending on the tropism of the serotype used. To determine the 

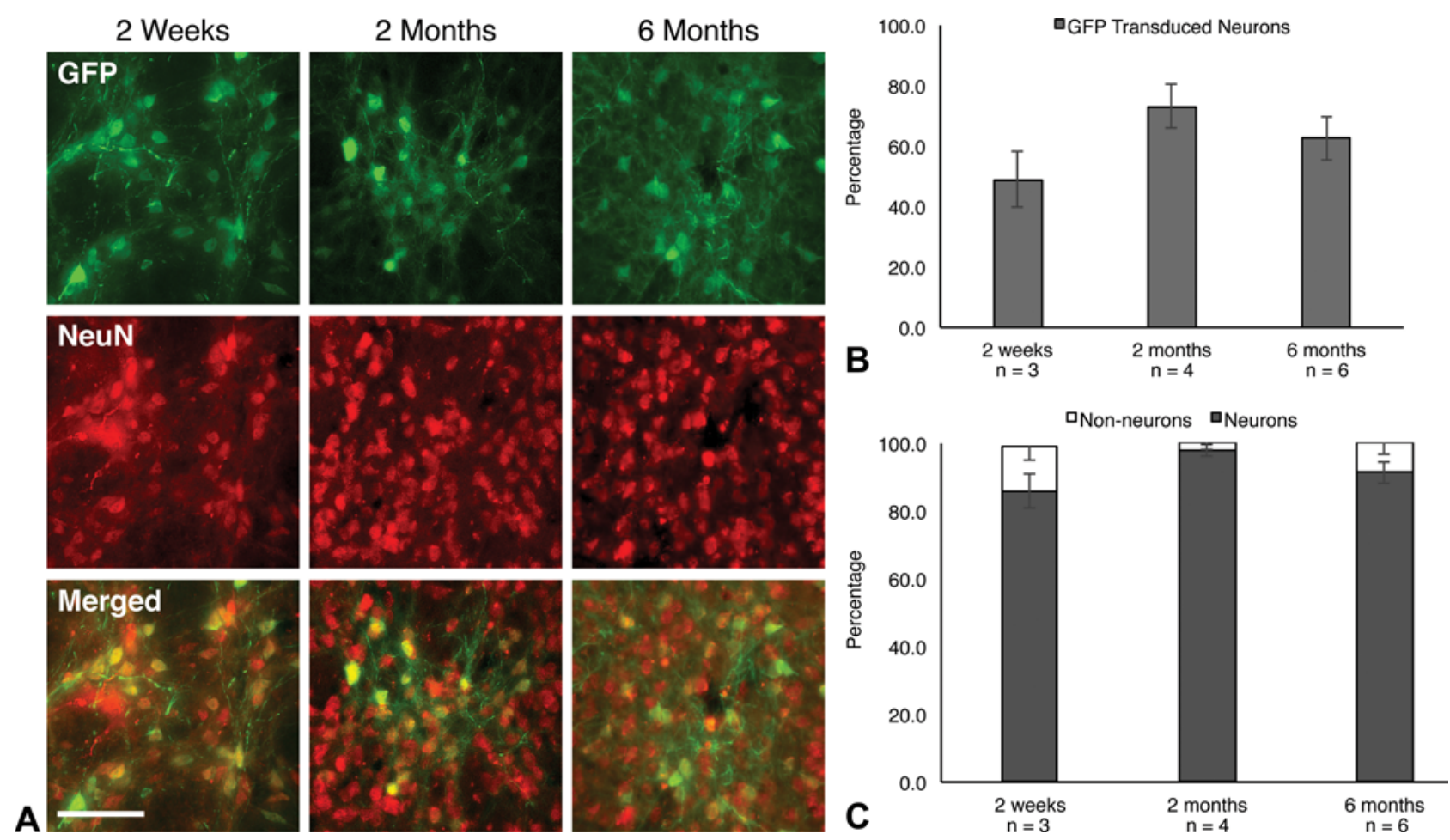

FIG. 2. MRgFUS facilitates stable, long-term GFP transduction. A: High-magnification immunostaining for GFP and NeuN reveals a dominantly neuronal population of GFP-transduced cells in the striatum. Bar $=50 \mu \mathrm{m}$. B: Bar graph showing that quantification of striatal GFP transduction is stable over time. GFP-positive neurons are expressed as a percentage of the total number of striatal neurons per $20 \mathrm{hpf}$ per animal (see Methods). C: Bar graph demonstrating that the MRgFUS-mediated GFP transduction is restricted mainly to neurons.

extent of peripheral gene expression, the heart, lung, and liver were harvested at the different time points following unilateral striatal MRgFUS and immunostained for GFP expression. While GFP was detected in the liver 2 weeks after sonication, no signal was detected at later time points (6 months and 16 months), consistent with a likely immune-mediated loss of gene expression as observed in other studies of foreign transgenes in this organ., ${ }^{3,23}$ Heart and lungs did not test positive for GFP at any time point (Fig. 4). As a negative control, we harvested these same organs from animals in which AAV1/2.GFP was delivered by direct infusion into striatum, with no evidence of gene expression in any organ.

\section{MRgFUS and Transitory Local Inflammatory Response}

The opening of the BBB, even when transient, could permit passage of various components from the blood stream into brain parenchyma. Previous studies have reported evidence of inflammation in the targeted brain soon after MRgFUS. No astrocytosis or microgliosis was detected in either sonicated or nonsonicated striatum at 2 weeks, 2 months, and 6 months after MRgFUS BBB disruption and AAV delivery (Fig. 5). No evidence of inflammatory response or tissue damage in the sonicated area was detected at any time point in both striatum and cortex (Fig. 5; Supplemental Figs. 3, 6, and 7). Given prior reports of transient inflammation following MRgFUS-mediated $\mathrm{BBB}$ disruption alone without delivering any agent to the brain, ${ }^{18,21}$ we performed striatal MRgFUS BBB opening alone in additional animals, killed at 3,24, and 48 hours, and confirmed (as expected) an increase in both Ibal and GFAP staining at these early time points in both striatum and cortex (Supplemental Figs. 8 and 9). Finally, while we did not conduct formal behavioral testing, regular gross observation revealed no evidence of abnormal behavior, and periodic monitoring of body weights and food intake showed no evidence of gross metabolic abnormality to suggest poor health.

\section{Discussion}

Minimally invasive or noninvasive therapies are increasingly attractive for diseases traditionally treated with invasive neurosurgical procedures. This is highlighted by the increasing application of radiosurgery for tumors and functional diseases, endovascular therapies for vascular diseases, and the recent interest in MRgFUS thalamotomy for essential tremor. Gene therapy in the nervous system remains experimental, but translation of human gene therapy has been led by the neurosurgical community with promising results from human studies. To date, all human CNS gene therapy studies have required direct surgical infusion due to the size of the viral particles and the presence of an intact BBB, which precludes efficient transfer of viral vectors from the blood to the brain. However, an advantage of direct infusion that would be lost with widespread delivery is the ability to manipulate only a defined population of neurons while avoiding altering off-target 


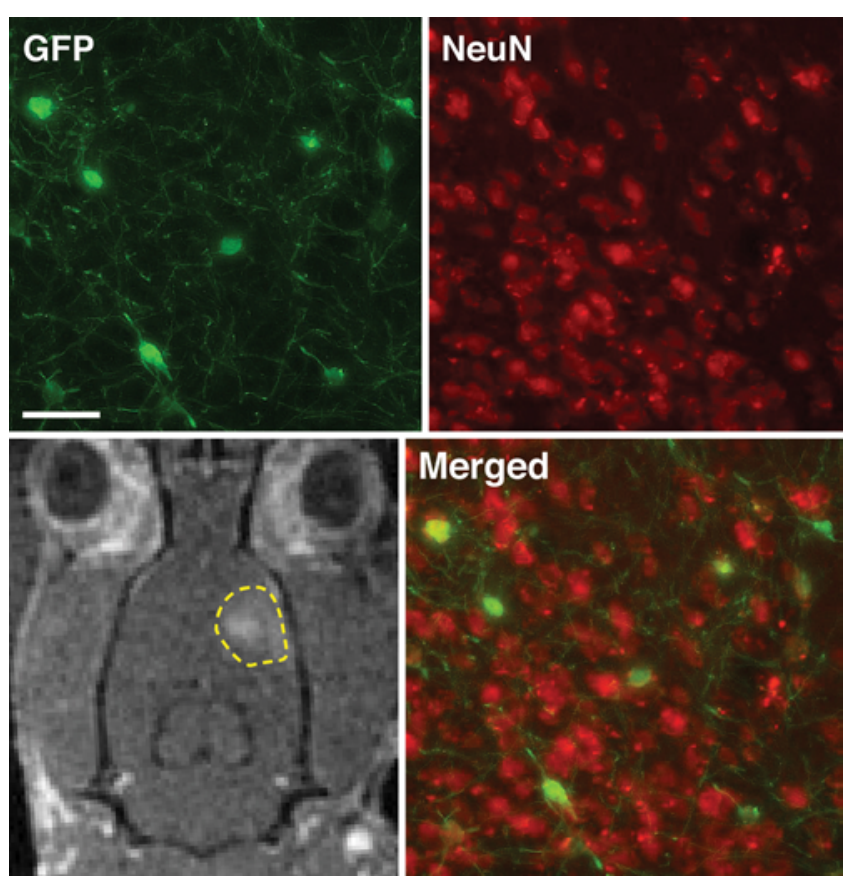

FIG. 3. Detection of striatal MRgFUS-facilitated AAV-mediated GFP transduction 16 months after sonication. Gd-DTPA-enhanced T1weighed images collected after sonication showed disruption of the BBB and Gd-DTPA extravasation in brain parenchyma (dashed line). Histological analysis of the brain harvested 16 months after sonication showed GFP transduction mostly in neurons. Bar $=50 \mu \mathrm{m}$.

cells, which could cause adverse or unintended effects. Previous studies have used MRgFUS BBB disruption to deliver viral vectors to the brain, but the efficiency, longterm stability of gene expression, and long-term safety of this approach remain unknown. Our results demonstrate that microbubble-facilitated MRgFUS successfully mediated passage of an AAV1/2 viral vector from the blood stream following intravenous administration into the targeted striatum without transduction of cells outside the zone of ultrasound delivery. This resulted in efficient and long-term GFP transduction within the targeted striatal neurons for more than 1 year and caused no obvious toxicity, neuronal loss, or long-term inflammatory response as measured by an absence of sustained astrocytic or microglial proliferation at various time points.

MRgFUS follows previous approaches to gene delivery through intravascular administration of viral vectors. The most common has been systemic use of an osmotic agent such as mannitol, which has been shown for many years to transiently open the BBB and has been used successfully in human patients for drug delivery. ${ }^{33,36-38}$ Several studies have evaluated the role of mannitol in facilitating global distribution, broad dispersion, and AAV-mediated gene expression in the brain..$^{11,12,26}$ In addition, intraarterial administration of mannitol with an AAV vector allowed limited BBB opening followed by target-specific gene expression. Real-time MRI visualization of Gd after BBB disruption was observed immediately upon intraarterial mannitol administration..$^{10}$ However, there are two caveats regarding the use of mannitol for transient BBB disruption. First, systemic administration of mannitol induces BBB opening throughout the brain. This could be highly desirable for widespread diseases, such as pediatric genetic disorders or infiltrative cancers, ${ }^{10,28,35}$ but could be problematic when delivering genes that could be therapeutic in one brain region but harmful in other areas, such as the glutamic acid decarboxylase gene for Parkinson's disease. ${ }^{8,19,22}$ Sitespecific delivery of mannitol via an intraarterial catheter could overcome this problem, but the variability in vascular supply to specific brain regions and the lower limit of the caliber of vessels that can currently be accessed could make reliable transduction of defined targets between individuals with limited off-target transduction more difficult.

One possible concern about MRgFUS BBB disruption is the potential for causing inflammation and tissue damage following brain exposure to the systemic immune system. This could be of particular concern when delivering a potential immunogen, such as a viral vector. Earlier studies in rodents have shown evidence of inflammatory reactions following FUS BBB disruption alone, without delivery of any agents. One report, using the same inflammatory markers that we used here, showed increased microglial activation several hours after BBB disruption, with an astrocytic response observed several days later. ${ }^{18} \mathrm{~A}$ recent study has more extensively examined both cellular and humoral mediators of inflammation from 1 to 24 hours following MRgFUS BBB disruption and reported evidence of a sterile inflammatory response. ${ }^{21}$ The goal of our study was to explore long-term consequences of MRgFUS delivery of gene therapy agents to the brain to determine the potential translational therapeutic relevance of this approach, so we did not examine inflammatory responses in such fine detail in the first 24-48 hours following delivery. Nonetheless, we did see some evidence of early microgliosis and astrocytosis between 3 and 48 hours following MRgFUS BBB disruption alone, consistent with these earlier reports. ${ }^{21}$ However, this appeared to resolve over a period of days, and was not evident at our 2-week through 6-month time points. We also saw no evidence of gross tissue damage or neuronal loss, nor any obvious behavioral abnormalities, to suggest significant toxicity from MRgFUS-mediated delivery of AAV vectors to the striatum under the conditions used in our study. It should be noted that immune reactions are generally more profound after subsequent exposures, and one potential advantage of gene therapy is the ability to provide long-term benefits from a single treatment. However, should more than one treatment become necessary for particular applications of MRgFUS BBB disruption, regardless of whether this is used for gene transduction or delivery of any other agent, the transient inflammatory reactions observed in both our study and earlier reports could represent a greater concern and should be studied carefully prior to considering clinical applications.

Another issue highlighted by our study is the problem of transduction of systemic organs with intravenous viral vector administration, even if MRgFUS restricts delivery within the brain. This could not only lead to production of potentially bioactive molecules from the gene of interest in undesirable organs, leading to adverse consequences, but also provoke inflammatory reactions that could result in organ tissue damage. In our study, a dose of $10^{9}$ 


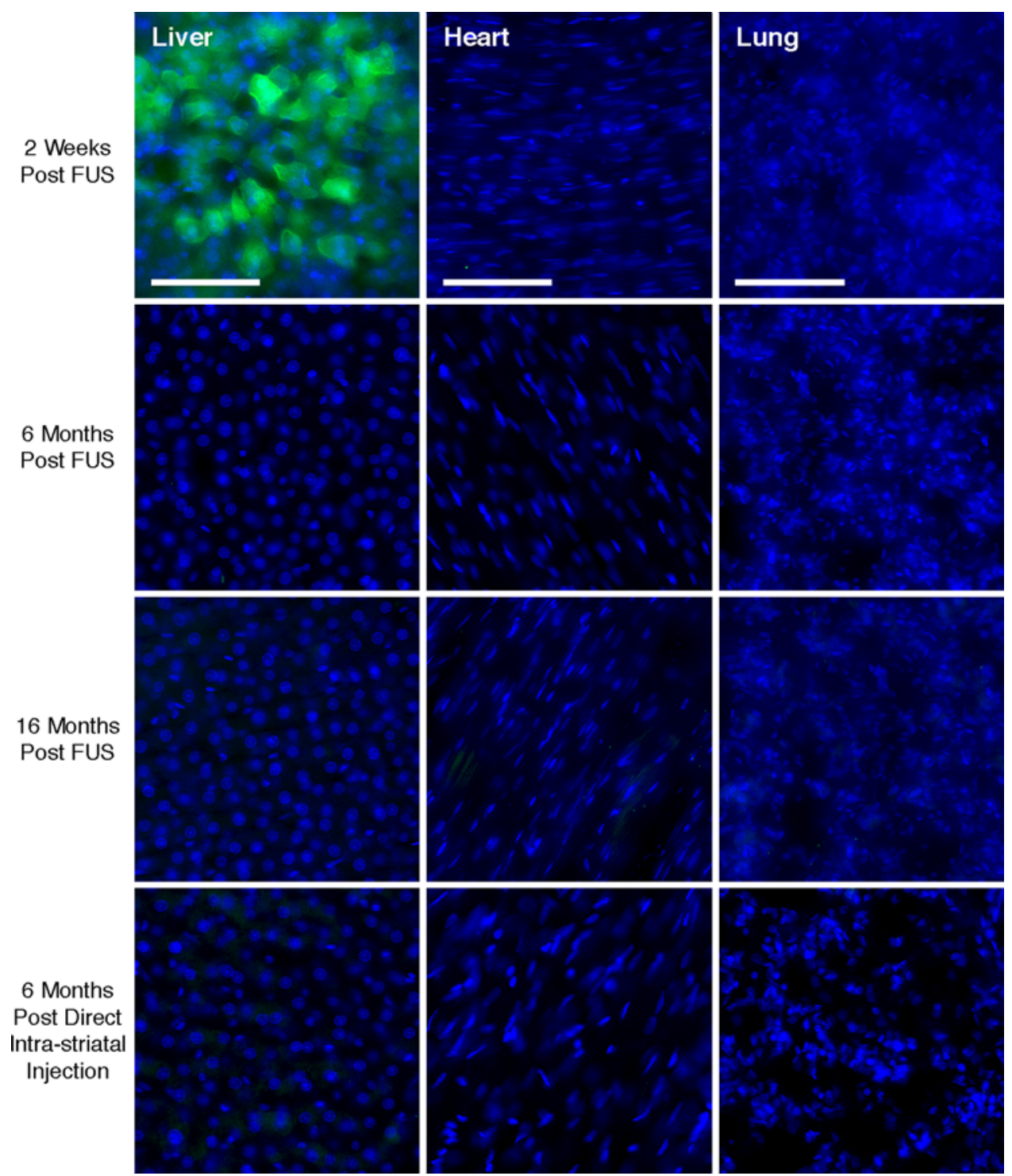

FIG. 4. MRgFUS-facilitated AAV-mediated gene delivery in peripheral organs is present in the short term but not the long term. Analysis of high-power immunofluorescent images of tissue collected from animals with MRgFUS with AAV1/2.GFP, and animals with AAV1/2.GFP stereotactically administered in the striatum shows no long-term GFP transgene expression in the liver, heart, and lungs. Bar $=100 \mu \mathrm{m}$. FUS $=$ MRgFUS.

$\mathrm{vg} / \mathrm{g}$ of AAV1/2.GFP administered via a tail vein catheter was sufficient to efficiently transduce the sonicated area in the brain. Under these conditions, we did not observe GFP expression in the heart or lungs at any time point. However, the liver revealed GFP expression at 2 weeks, which was lost at later time points. This could represent either a loss of gene expression, loss of cells, or both, and extensive analyses of the causes of this observation in the liver are beyond the scope of our current study. However, previous reports have examined the phenomenon of loss of expression in peripheral organs several weeks after AAV transduction. Often this appears to be an immune-mediated effect. ${ }^{3,4,23}$ While GFP is a foreign gene, which could provoke immune responses that might not be observed with delivery of genes encoding native proteins, immune reactions against the AAV vector used to transduce the cells can also cause loss of expression even after transduction has successfully occurred. ${ }^{3,23}$ In fact, the loss of GFP expression in the liver several weeks following gene delivery without loss of expression in the brain further supports the likelihood that restoration of brain immune privilege following closure of the BBB was achieved prior to onset of GFP expression. Nonetheless, to both limit off-target effects of gene expression in peripheral organs and prevent possible immune-mediated tissue injury, development of methods to restrict viral vector transduction of peripheral organs following intravenous delivery may be very important for successful clinical translation of MRgFUS-mediated gene delivery for many CNS disorders.

There are several limitations of our study, which could be addressed in the future. As a pilot study to determine the safety and stability of gene expression, we included a 


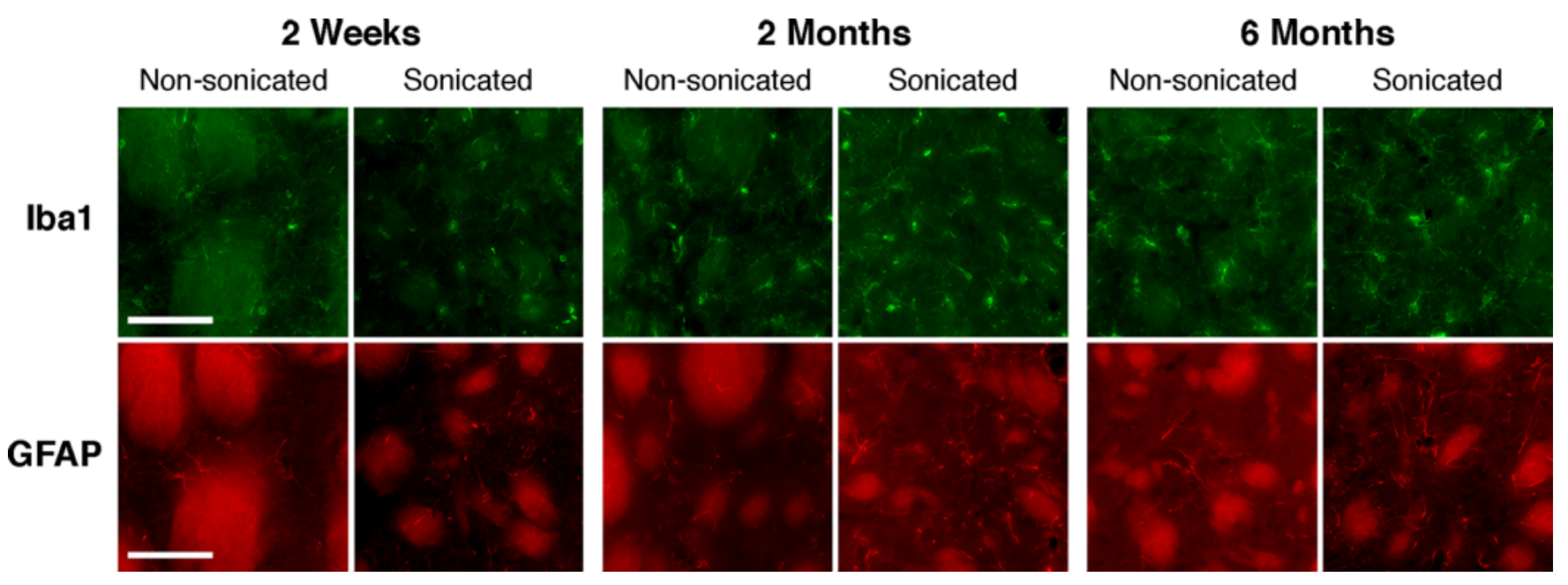

FIG. 5. No evidence of long-term inflammatory response induced by unilateral striatal MRgFUS-mediated gene transfer to the brain. Both Iba1 (microglia marker) and GFAP (astrocytic marker) returned to baseline levels by week 2, suggesting that local inflammatory response is transitory. Bar $=50 \mu \mathrm{m}$.

relatively small number of animals in each group, particularly at the 16-month time point, although there was consistency among animals at each time point. Therefore, more infrequent complications may not have been detected here. Furthermore, the rodent device that we have employed uses a single transducer element, so the area of focus is rather large compared with what might be achieved with an array of transducers, such as the system currently in use for human lesioning. The rodent immune system also may not accurately reflect the human condition, and ultimately it will be important to replicate our findings in other species to gain sufficient confidence prior to considering human translation. It would also be interesting to compare the relative transduction efficiencies of direct injection and systemic delivery from MRgFUS to the same structures, but the current study was not designed to address this. Finally, while we did not observe significant long-term inflammation, cell death, or loss of expression, it is possible that use of other AAV serotypes or different viral vectors or other transgenes, as well as different ultrasound parameters, could lead to different results. While our results do not guarantee that all parameters and viral vectors will be equivalent without additional testing, our data do support the potential of MRgFUS as a safe and efficient means for noninvasive, stable, focal gene delivery in the mammalian brain.

\section{Conclusions}

Our study demonstrates that BBB disruption using MRgFUS can be a safe and efficient method for sitespecific delivery of viral vectors to the brain, suggesting the potential for noninvasive human gene therapy. While further study is necessary, the long-term safety and stability of gene expression reported here provides a framework for future development of this approach. Since direct infusion of AAV vectors has been safely applied to many human patients for a variety of diseases, and at least one human clinical device was recently FDA approved to perform MRgFUS thalamotomy for essential tremor, all nec- essary technology is currently available for translation of our findings into human studies. Further work is needed to address remaining questions, including the applicability to the primate brain and potential safety issues from peripheral gene expression and central inflammation, depending on the specific application. Nonetheless, our long-term safety and gene expression data support the continued development of this approach as a potentially viable future option for noninvasive focal CNS gene therapy.

\section{Acknowledgments}

We kindly thank Jojo Borja and Jonathan Dyke, PhD, for providing technical MRI assistance. This research was funded by a grant from the JPB Foundation (M.G.K.).

\section{References}

1. Abbott NJ, Patabendige AA, Dolman DE, Yusof SR, Begley DJ: Structure and function of the blood-brain barrier. Neurobiol Dis 37:13-25, 2010

2. Alonso A, Reinz E, Leuchs B, Kleinschmidt J, Fatar M, Geers B, et al: Focal delivery of AAV2/1-transgenes into the rat brain by localized ultrasound-induced BBB opening. Mol Ther Nucleic Acids 2:e73, 2013

3. Bell P, Gao G, Haskins ME, Wang L, Sleeper M, Wang H, et al: Evaluation of adeno-associated viral vectors for liverdirected gene transfer in dogs. Hum Gene Ther 22:985-997, 2011

4. Breous E, Somanathan S, Bell P, Wilson JM: Inflammation promotes the loss of adeno-associated virus-mediated transgene expression in mouse liver. Gastroenterology 141:348357, 357.e1-357.e3, 2011

5. Carty N, Lee D, Dickey C, Ceballos-Diaz C, Jansen-West K, Golde TE, et al: Convection-enhanced delivery and systemic mannitol increase gene product distribution of AAV vectors 5,8 , and 9 and increase gene product in the adult mouse brain. J Neurosci Methods 194:144-153, 2010

6. Elias WJ, Huss D, Voss T, Loomba J, Khaled M, Zadicario E, et al: A pilot study of focused ultrasound thalamotomy for essential tremor. N Engl J Med 369:640-648, 2013

7. Elias WJ, Lipsman N, Ondo WG, Ghanouni P, Kim YG, Lee $\mathrm{W}$, et al: A randomized trial of focused ultrasound thalamotomy for essential tremor. N Engl J Med 375:730-739, 2016 
8. Feigin A, Kaplitt MG, Tang C, Lin T, Mattis P, Dhawan V, et al: Modulation of metabolic brain networks after subthalamic gene therapy for Parkinson's disease. Proc Natl Acad Sci U S A 104:19559-19564, 2007

9. Fiandaca MS, Varenika V, Eberling J, McKnight T, Bringas J, Pivirotto P, et al: Real-time MR imaging of adeno-associated viral vector delivery to the primate brain. Neuroimage 47 (Suppl 2):T27-T35, 2009

10. Foley CP, Rubin DG, Santillan A, Sondhi D, Dyke JP, Crystal RG, et al: Intra-arterial delivery of AAV vectors to the mouse brain after mannitol mediated blood brain barrier disruption. J Control Release 196:71-78, 2014

11. Fu H, Kang L, Jennings JS, Moy SS, Perez A, Dirosario J, et al: Significantly increased lifespan and improved behavioral performances by rAAV gene delivery in adult mucopolysaccharidosis IIIB mice. Gene Ther 14:1065-1077, 2007

12. Fu H, Muenzer J, Samulski RJ, Breese G, Sifford J, Zeng X, et al: Self-complementary adeno-associated virus serotype 2 vector: global distribution and broad dispersion of AAVmediated transgene expression in mouse brain. Mol Ther 8:911-917, 2003

13. Hsu PH, Wei KC, Huang CY, Wen CJ, Yen TC, Liu CL, et al: Noninvasive and targeted gene delivery into the brain using microbubble-facilitated focused ultrasound. PLoS One 8:e57682, 2013

14. Huang Q, Deng J, Wang F, Chen S, Liu Y, Wang Z, et al: Targeted gene delivery to the mouse brain by MRI-guided focused ultrasound-induced blood-brain barrier disruption. Exp Neurol 233:350-356, 2012

15. Hynynen K: Focused ultrasound for blood-brain disruption and delivery of therapeutic molecules into the brain. Expert Opin Drug Deliv 4:27-35, 2007

16. Hynynen K, McDannold N, Vykhodtseva N, Jolesz FA: Noninvasive MR imaging-guided focal opening of the bloodbrain barrier in rabbits. Radiology 220:640-646, 2001

17. Jeanmonod D, Werner B, Morel A, Michels L, Zadicario E, Schiff G, et al: Transcranial magnetic resonance imagingguided focused ultrasound: noninvasive central lateral thalamotomy for chronic neuropathic pain. Neurosurg Focus 32(1):E1, 2012

18. Jordão JF, Thévenot E, Markham-Coultes K, Scarcelli T, Weng YQ, Xhima K, et al: Amyloid- $\beta$ plaque reduction, endogenous antibody delivery and glial activation by braintargeted, transcranial focused ultrasound. Exp Neurol 248:16-29, 2013

19. Kaplitt MG, Feigin A, Tang C, Fitzsimons HL, Mattis P, Lawlor PA, et al: Safety and tolerability of gene therapy with an adeno-associated virus (AAV) borne GAD gene for Parkinson's disease: an open label, phase I trial. Lancet 369:2097-2105, 2007

20. Kaplitt MG, Leone P, Samulski RJ, Xiao X, Pfaff DW, O'Malley KL, et al: Long-term gene expression and phenotypic correction using adeno-associated virus vectors in the mammalian brain. Nat Genet 8:148-154, 1994

21. Kovacs ZI, Kim S, Jikaria N, Qureshi F, Milo B, Lewis BK, et al: Disrupting the blood-brain barrier by focused ultrasound induces sterile inflammation. Proc Natl Acad Sci U S A 114:E75-E84, 2017

22. LeWitt PA, Rezai AR, Leehey MA, Ojemann SG, Flaherty AW, Eskandar EN, et al: AAV2-GAD gene therapy for advanced Parkinson's disease: a double-blind, sham-surgery controlled, randomised trial. Lancet Neurol 10:309-319, 2011

23. Manno CS, Pierce GF, Arruda VR, Glader B, Ragni M, Rasko JJ, et al: Successful transduction of liver in hemophilia by AAV-factor IX and limitations imposed by the host immune response. Nat Med 12:342-347, 2006 (Erratum in Nat Med 12:592, 2006)

24. Marks WJ Jr, Bartus RT, Siffert J, Davis CS, Lozano A, Bou- lis N, et al: Gene delivery of AAV2-neurturin for Parkinson's disease: a double-blind, randomised, controlled trial. Lancet Neurol 9:1164-1172, 2010

25. Marks WJ Jr, Ostrem JL, Verhagen L, Starr PA, Larson PS, Bakay RA, et al: Safety and tolerability of intraputaminal delivery of CERE-120 (adeno-associated virus serotype 2-neurturin) to patients with idiopathic Parkinson's disease: an open-label, phase I trial. Lancet Neurol 7:400-408, 2008

26. Mastakov MY, Baer K, Xu R, Fitzsimons H, During MJ: Combined injection of rAAV with mannitol enhances gene expression in the rat brain. Mol Ther 3:225-232, 2001

27. McCaffrey G, Davis TP: Physiology and pathophysiology of the blood-brain barrier: P-glycoprotein and occludin trafficking as therapeutic targets to optimize central nervous system drug delivery. J Investig Med 60:1131-1140, 2012

28. McCarty DM, DiRosario J, Gulaid K, Muenzer J, Fu H: Mannitol-facilitated CNS entry of rAAV2 vector significantly delayed the neurological disease progression in MPS IIIB mice. Gene Ther 16:1340-1352, 2009

29. McDannold N, Vykhodtseva N, Raymond S, Jolesz FA, Hynynen K: MRI-guided targeted blood-brain barrier disruption with focused ultrasound: histological findings in rabbits. Ultrasound Med Biol 31:1527-1537, 2005

30. Mingozzi F, High KA: Immune responses to AAV in clinical trials. Curr Gene Ther 11:321-330, 2011

31. Morgenstern PF, Marongiu R, Musatov SA, Kaplitt MG: Adeno-associated viral gene delivery in neurodegenerative disease. Methods Mol Biol 793:443-455, 2011

32. Nance E, Timbie K, Miller GW, Song J, Louttit C, Klibanov AL, et al: Non-invasive delivery of stealth, brain-penetrating nanoparticles across the blood-brain barrier using MRI-guided focused ultrasound. J Control Release 189:123-132, 2014

33. Neuwelt EA, Balaban E, Diehl J, Hill S, Frenkel E: Successful treatment of primary central nervous system lymphomas with chemotherapy after osmotic blood-brain barrier opening. Neurosurgery 12:662-671, 1983

34. Neuwelt EA, Barnett PA, McCormick CI, Frenkel EP, Minna JD: Osmotic blood-brain barrier modification: monoclonal antibody, albumin, and methotrexate delivery to cerebrospinal fluid and brain. Neurosurgery 17:419-423, 1985

35. Neuwelt EA, Dahlborg SA: Chemotherapy administered in conjunction with osmotic blood-brain barrier modification in patients with brain metastases. J Neurooncol 4:195-207, 1987

36. Neuwelt EA, Diehl JT, Vu LH, Hill SA, Michael AJ, Frenkel EP: Monitoring of methotrexate delivery in patients with malignant brain tumors after osmotic blood-brain barrier disruption. Ann Intern Med 94:449-454, 1981

37. Neuwelt EA, Goldman DL, Dahlborg SA, Crossen J, Ramsey F, Roman-Goldstein S, et al: Primary CNS lymphoma treated with osmotic blood-brain barrier disruption: prolonged survival and preservation of cognitive function. J Clin Oncol 9:1580-1590, 1991

38. Neuwelt EA, Hill SA, Frenkel EP: Osmotic blood-brain barrier modification and combination chemotherapy: concurrent tumor regression in areas of barrier opening and progression in brain regions distant to barrier opening. Neurosurgery 15:362-366, 1984

39. Neuwelt EA, Maravilla KR, Frenkel EP, Rapaport SI, Hill SA, Barnett PA: Osmotic blood-brain barrier disruption. Computerized tomographic monitoring of chemotherapeutic agent delivery. J Clin Invest 64:684-688, 1979

40. Rafii MS, Baumann TL, Bakay RA, Ostrove JM, Siffert J, Fleisher AS, et al: A phasel study of stereotactic gene delivery of AAV2-NGF for Alzheimer's disease. Alzheimers Dement 10:571-581, 2014

41. Salegio EA, Samaranch L, Kells AP, Forsayeth J, Bankiewicz $\mathrm{K}$ : Guided delivery of adeno-associated viral vectors into the primate brain. Adv Drug Deliv Rev 64:598-604, 2012 
42. Schuster DJ, Dykstra JA, Riedl MS, Kitto KF, Belur LR, McIvor RS, et al: Biodistribution of adeno-associated virus serotype 9 (AAV9) vector after intrathecal and intravenous delivery in mouse. Front Neuroanat 8:42, 2014

43. Thévenot E, Jordão JF, O'Reilly MA, Markham K, Weng YQ, Foust KD, et al: Targeted delivery of self-complementary adeno-associated virus serotype 9 to the brain, using magnetic resonance imaging-guided focused ultrasound. Hum Gene Ther 23:1144-1155, 2012

44. Treat LH, McDannold N, Vykhodtseva N, Zhang Y, Tam K, Hynynen K: Targeted delivery of doxorubicin to the rat brain at therapeutic levels using MRI-guided focused ultrasound. Int J Cancer 121:901-907, 2007

45. Treat LH, McDannold N, Zhang Y, Vykhodtseva N, Hynynen $\mathrm{K}$ : Improved anti-tumor effect of liposomal doxorubicin after targeted blood-brain barrier disruption by MRI-guided focused ultrasound in rat glioma. Ultrasound Med Biol 38:1716-1725, 2012

46. Tuszynski MH, Thal L, Pay M, Salmon DP, U HS, Bakay $\mathrm{R}$, et al: A phase 1 clinical trial of nerve growth factor gene therapy for Alzheimer disease. Nat Med 11:551-555, 2005

47. Tuszynski MH, Yang JH, Barba D, U HS, Bakay RA, Pay MM, et al: Nerve growth factor gene therapy: activation of neuronal responses in Alzheimer disease. JAMA Neurol 72:1139-1147, 2015

48. Wang L, Cao O, Swalm B, Dobrzynski E, Mingozzi F, Herzog RW: Major role of local immune responses in antibody formation to factor IX in AAV gene transfer. Gene Ther 12:1453-1464, 2005

49. Wang S, Kugelman T, Buch A, Herman M, Han Y, Karakatsani ME, et al: Non-invasive, focused ultrasound-facilitated gene delivery for optogenetics. Sci Rep 7:39955, 2017

50. Wang S, Olumolade OO, Sun T, Samiotaki G, Konofagou EE: Noninvasive, neuron-specific gene therapy can be facilitated by focused ultrasound and recombinant adeno-associated virus. Gene Ther 22:104-110, 2015

\section{Disclosures}

The authors report no conflict of interest concerning the materials or methods used in this study or the findings specified in this paper.

\section{Author Contributions}

Conception and design: Kaplitt, Stavarache, Ballon. Acquisition of data: all authors. Analysis and interpretation of data: Kaplitt, Stavarache, Petersen, Ballon. Drafting the article: Kaplitt, Stavarache. Critically revising the article: Kaplitt, Stavarache. Reviewed submitted version of manuscript: all authors. Approved the final version of the manuscript on behalf of all authors: Kaplitt. Statistical analysis: Kaplitt, Stavarache. Administrative/technical/material support: Kaplitt. Study supervision: Kaplitt, Stavarache.

\section{Supplemental Information \\ Videos \\ Video 1. https://vimeo.com/242943981.}

\section{Online-Only Content}

Supplemental material is available with the online version of the article.

Supplemental Figs. 1-9. https://thejns.org/doi/suppl/10.3171/ 2017.8.JNS17790.

\section{Previous Presentations}

Portions of this work were presented in a nanosymposium at the Society for Neuroscience in San Diego, California, on November $16,2016$.

\section{Correspondence}

Michael G. Kaplitt: Weill Cornell Medical College, New York, NY.mik2002@med.cornell.edu. 